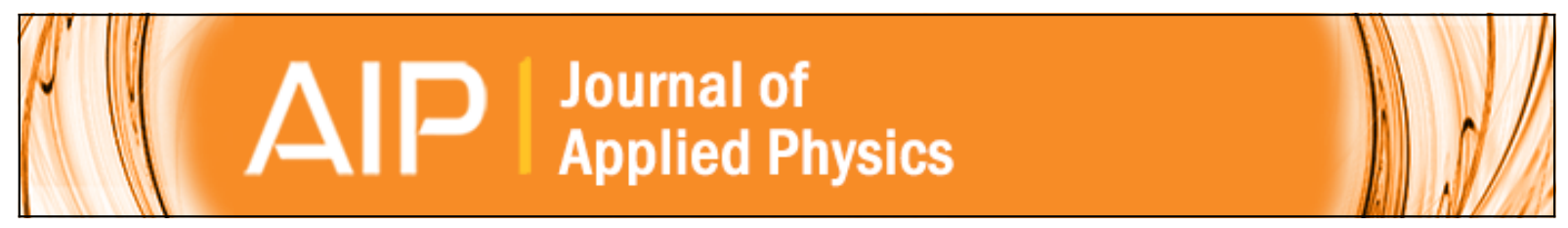

\title{
Minority-carrier effects in poly-phenylenevinylene as studied by electrical characterization
}

P. Stallinga, H. L. Gomes, H. Rost, A. B. Holmes, M. G. Harrison, and R. H. Friend

Citation: Journal of Applied Physics 89, 1713 (2001); doi: 10.1063/1.1334634

View online: http://dx.doi.org/10.1063/1.1334634

View Table of Contents: http://scitation.aip.org/content/aip/journal/jap/89/3?ver=pdfcov

Published by the AIP Publishing

\section{Articles you may be interested in}

Importance of minority carrier response in accurate characterization of Ge metal-insulator-semiconductor interface traps

J. Appl. Phys. 106, 044506 (2009); 10.1063/1.3204025

Effects of thermal annealing on deep-level defects and minority-carrier electron diffusion length in Be-doped InGaAsN

J. Appl. Phys. 97, 073702 (2005); 10.1063/1.1871334

Electrical characterization of strained $\mathrm{Si} / \mathrm{Si}$ Ge wafers using transient capacitance measurements Appl. Phys. Lett. 86, 122111 (2005); 10.1063/1.1891303

Observed trapping of minority-carrier electrons in p -type GaAsN during deep-level transient spectroscopy measurement

Appl. Phys. Lett. 86, 072109 (2005); 10.1063/1.1865328

Majority- and minority-carrier traps in Te-doped AllnP

Appl. Phys. Lett. 74, 284 (1999); 10.1063/1.123000

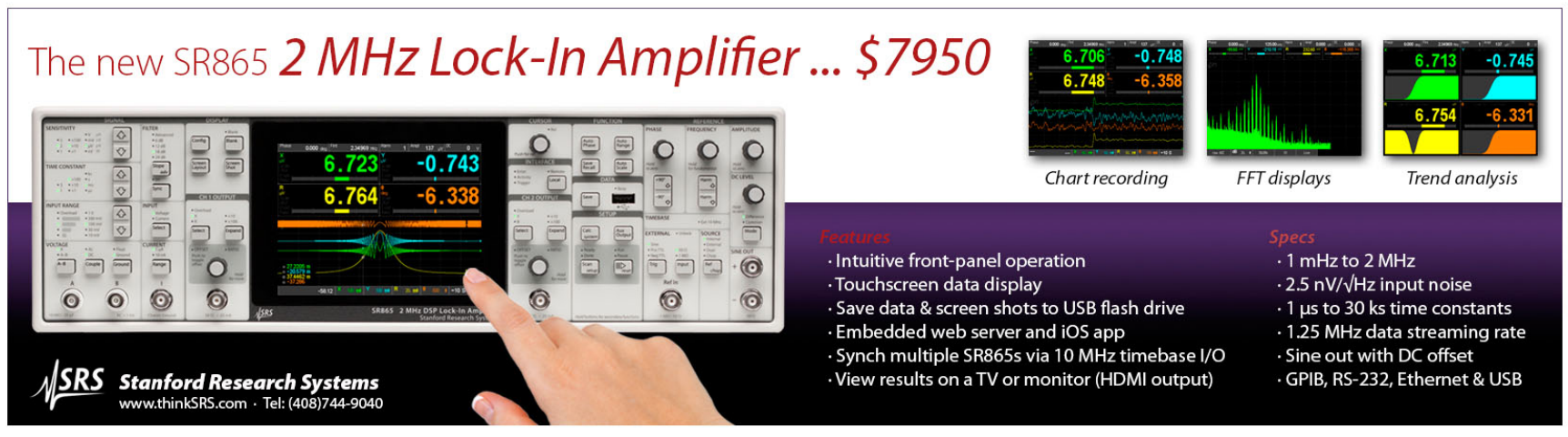




\title{
Minority-carrier effects in poly-phenylenevinylene as studied by electrical characterization
}

\author{
P. Stallinga ${ }^{a}$ and H. L. Gomes \\ Universidade do Algarve, UCEH, Campus de Gambelas, 8000 Faro, Portugal \\ H. Rost and A. B. Holmes \\ Melville Laboratory for Polymer Synthesis, Pembroke Street, Cambridge CB2 3RA, United Kingdom \\ M. G. Harrison and R. H. Friend \\ Cavendish Laboratory, Madingley Road, Cambridge CB3 OHE, United Kingdom
}

(Received 12 July 1999; accepted for publication 27 October 2000)

\begin{abstract}
Electrical measurements have been performed on poly[2-methoxy, 5 ethyl ( $2^{\prime}$ hexyloxy) paraphenylenevinylene] in a $p n$ junction with silicon. These included current-voltage measurements, capacitance-voltage measurements, capacitance-transient spectroscopy, and admittance spectroscopy. The measurements show evidence for large minority-carrier injection into the polymer possibly enabled by interface states for which evidence is also found. The shallow acceptor level depth $(0.12 \mathrm{eV})$ and four deep trap level activation energies $(0.30$ and $1.0 \mathrm{eV}$ majority-carrier type; 0.48 and $1.3 \mathrm{eV}$ minority-carrier type) are found. Another trap that is visible at room temperature has point-defect nature. (C) 2001 American Institute of Physics.
\end{abstract}

[DOI: $10.1063 / 1.1334634]$

\section{INTRODUCTION}

Conjugated polymers have structural flexibility and their weak intermolecular bonding leads to a variety of possible structures that are unmatched by any other class of materials. ${ }^{1-3}$ These properties are being exploited successfully in the fabrication of light-emitting diodes (LEDs) in all colors of the visible spectrum, including the hard-soughtafter blue LED. ${ }^{4}$ In fact, full color displays ${ }^{5}$ using three primary colors close to the Commission Internationale de l'Eclairage (CIE) standards and color scanners ${ }^{6}$ have already been fabricated from organic materials.

Although it has been shown that minority carriers play a major role in determining the efficiency of light emission in LEDs, there is scarce knowledge of their properties. Evidence has been found for the existence of electron traps that are responsible for the quenching of the electroluminescence, ${ }^{7,8}$ but so far little is known about the nature of these states and on the parameters characterizing them.

Extracting unambiguous information about minoritycarrier states using common LED structures is a difficult task. These devices are normally fabricated using reactive metals such as calcium, which chemically reacts with the polymers, ${ }^{9}$ and indium tin oxide (ITO) which recently has also been found to be an unstable electrode. ${ }^{10}$ The metalpolymer interfaces are ill-defined and complex and considerable care has to be taken to ensure a meaningful interpretation of the measurements.

In this study we used a device structure where the electrodes do not chemically interact with the polymer. The structure is an $n^{+}$-silicon/polymer/gold device where the highly doped silicon acts as an electron injector and the gold

\footnotetext{
${ }^{a)}$ Electronic mail: pjotr@ualg.pt
}

as a hole injector. Furthermore, the structure may be of technological importance when the polymer is integrated in monolithic silicon circuits. LED structures using doped silicon as hole-injection material have been previously demonstrated by Parker and Kim. ${ }^{11}$

Using this structure, we have undertaken an extensive series of measurements in an effort to extract information about the properties of gap states and carrier injection. The techniques which we have used included standard $I-V$ measurements, steady-state capacitance, and conductance methods as well as deep-level transient-spectroscopy (DLTS) measurements and thermally stimulated current (TSC). Previous DLTS studies in polymers have made use of Schottky barrier devices ${ }^{12}$ which are single-carrier devices and are therefore not suitable for probing minority-carrier traps. Campbell et al. have also applied DLTS techniques to ITO/ $\mathrm{PPV} / \mathrm{Al}$ structures $(\mathrm{PPV}=$ poly-paraphenylenevinylene $)$ and reported nonexponential transients. ${ }^{13}$ In the current study we show how we can probe both majority and minority traps by using silicon as the cathode, and find discrete energy levels. We also show the evidence for a thin isolating interfacial layer that influences the minority-carrier injection.

\section{ENERGY DIAGRAM}

The knowledge about the band structure of \{poly[2methoxy, 5 ethyl $\left(2^{\prime}\right.$ hexyloxy) paraphenylenevinylene]\} (MEH-PPV) can be summarized as: Electron affinity [energy from vacuum to the bottom of the conduction band or lowest unoccupied molecular orbit (LUMO)] is $2.5 \mathrm{eV}$ and the band gap [from the LUMO to the highest occupied molecular orbit (HOMO) the equivalent of the valence band] is also 2.5 $\mathrm{eV} .{ }^{14} \mathrm{~A} p$-type MEH-PPV polymer is therefore expected to form a Schottky barrier with a depletion region when brought into contact with metals such as aluminum (work 


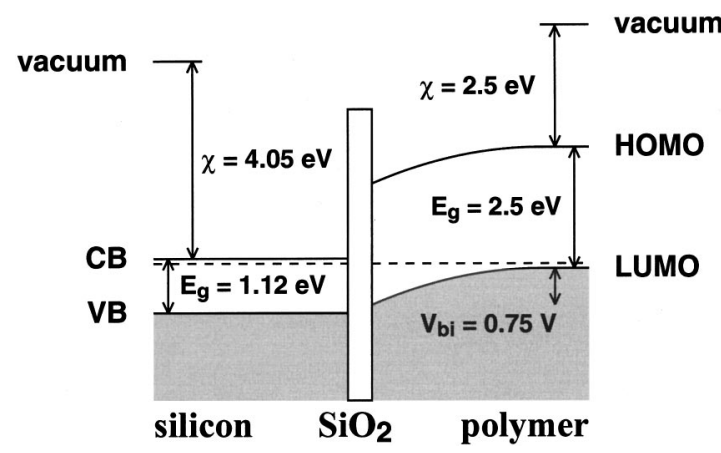

FIG. 1. Expected energy diagram of an $n$-type silicon/PPV device with a thin insulating layer (over which no voltage drop occurs). On basis of the electron affinities and band gaps of silicon (Ref. 15) and PPV (Ref. 14), a rectifying contact is expected with a built-in voltage of $0.75 \mathrm{~V}$.

function only $4.3 \mathrm{eV}$ ), but to form ohmic contacts with, for instance, gold (with a work function of $5.1 \mathrm{eV}$ ). With the latter, an accumulated region is formed with an increased density of free carriers at the interface. When the polymer is deposited on top of heavily doped $n$-type silicon [electron affinity: $4.05 \mathrm{eV}$, band gap $1.15 \mathrm{eV}$ (Ref. 15)] the behavior is expected to be very similar to the aluminum-polymer interface with a built-in voltage of $\sim 0.75 \mathrm{~V}$ (calculated from the difference in Fermi level positions in the silicon and polymer, which are expected to be close, say $0.1 \mathrm{eV}$, to the conduction band and valence band, respectively).

The formation of a thin, insulating oxide on top of the silicon is unavoidable when, as in our case, the deposition is done at ambient conditions. This results in so-called metalinsulator-semiconductor (MIS) tunnel diodes as described by Card and Rhoderick. ${ }^{16}$ The combined expected structure of the device is shown in Fig. 1.

The presence of an interfacial layer provides an extra degree of freedom to the energy diagram; a voltage drop in the insulating layer allows a shift of the band structure of the metal relative to the semiconductor. Also interface states (caused by impurities or discontinuities at the interface) can have a pronounced effect on the device; charged interface states can cause a voltage drop across the interface which can produce effects similar to a difference in work function of the two joined materials, also, a large number of interface states can pin the Fermi level at a certain energy. They can thus, for instance, force the interface to be inverted (an abundance of minority-carriers) and make the minority current dominate over the majority-carrier current.

When a device is inverted at zero bias the minoritycarrier current will be a substantial fraction of the total current at small biases. When the voltage is increased, this ratio eventually drops. ${ }^{17,18}$ The minority-carrier current will decrease (or grow less rapidly), while the majority-carrier current continues to increase. The net effect is the formation of a plateau of reduced voltage dependence of the current in the forward bias $I-V$ (current-voltage) curve until the minoritycarrier current has died out and the device has become majority-carrier type. This plateau can cause the device to have inverted rectification over a certain voltage range; the reverse-bias current can be larger than the forward-bias current.

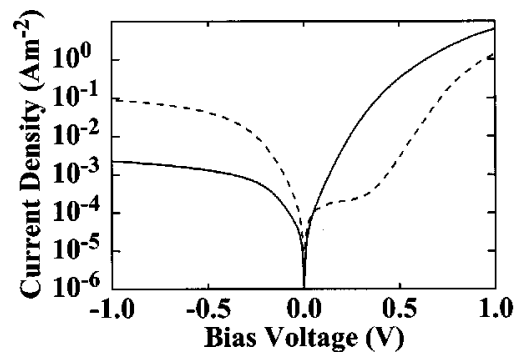

FIG. 2. Typical $I-V$ curves at $300 \mathrm{~K}$ after freshly mounting the sample from air into vacuum (dashed line) and after two weeks in vacuum (solid curve). The $I-V$ rectification ratio which was initialy very low increases to over 1000. Also, the plateau in the forward current, that is indicative for a substantial minority-carrier current, is removed. The device has been converted from minority-carrier type to majority-carrier type.

\section{EXPERIMENT}

The films of MEH-PPV ( $1 \mu \mathrm{m}$ thick) were deposited by spin coating onto $n^{+}$silicon substrates with a bulk resistivity of $0.1 \Omega \mathrm{cm}$. Prior to deposition, the silicon substrates were cleansed with diluted HF to remove any residual oxide layer and were rinsed several times in ultrapure water. Before the spin coating, the samples have been very briefly exposed to air and we estimate that this has caused an oxide layer on the silicon with a thickness of the order of $30 \AA$. After spin coating the films were immediately loaded into a turbopumped evaporator and gold was evaporated through a mask to form an array of circular electrodes ( $2 \mathrm{~mm}$ in diameter). For the measurements the devices were mounted in a temperature controlled sample holder located inside a steel chamber evacuated to less than $10^{-5}$ mbar. Small-signal admittance measurements over the range $50 \mathrm{~Hz}-1 \mathrm{MHz}$ were carried out with a Fluke PM 6306 RCL meter. The dc $I-V$ curves were recorded with a Keithley 487 picoammeter/ voltage source. The sample temperature was varied in the range $100-300 \mathrm{~K}$ and measured with a chromel-alumel thermocouple placed on the substrate close to the devices. All the measurements were carried out in the dark. From the fabrication process until the characterization, the samples have been in ambient (albeit dark) environments for some days. Prior to the measurements discussed here, the sample has undergone a heat treatment at $115^{\circ} \mathrm{C}$ in vacuum.

\section{CURRENT-VOLTAGE MEASUREMENTS}

Figure 2 shows a typical $I-V$ curve of the MEH-PPV/ $n^{+}$-silicon structure. The original scan, as taken freshly after mounting the sample into vacuum, shows some interesting features. The first thing to note is that the current densities are very high; the current is not much limited by the oxide layer. Second, the rectification ratio is very poor, only a factor 16 is achieved between the reverse and the forward current at $|1 \mathrm{~V}|$. Furthermore, the reverse current saturates, as predicted by thermionic emission theory (Bethe, see Sze for a comprehensive summary of dc conductance theories ${ }^{15}$ ). Finally, in the forward current there is a large plateau from 0 to $0.3-0.4 \mathrm{eV} \mathrm{V}$, as described in Sec. II. For small voltages, the $I-V$ characteristic is even inverted. This $I-V$ plot very much resembles the minority-carrier-MIS-tunnel diodes as 
described by Green and Shewchun ${ }^{19}$ and can be described in the following way: the MEH-PPV surface is in inversion under reverse and small forward biases. (This inversion might be caused by the presence of interface states which can pin the Fermi level in the top half of the band gap at the interface as described in the previous section.) As the bias is increased the device goes from inversion to depletion and eventually to accumulation. In inversion, the major component of the current is the flow of electrons from the silicon to the conduction band of the polymer. When the voltage is increased, the ratio minority-carrier current to majority carrier current eventually drops. ${ }^{17,18}$

Figure 2 also shows the $I-V$ characteristics after placing the device two weeks in vacuum. The rectification ratio at $\pm 1 \mathrm{~V}$ has soared to 2800 and the plateau in the forward current has nearly disappeared. The device behaves like a normal diode now, namely a device of nonequilibrium majority-carrier type. Since the silicon-oxide layer is stable under these conditions, the interface states must have changed or have been removed by the persistent pumping. This would then remove the voltage drop or the Fermi level pinning and therefore the inversion layer.

\section{AC CHARACTERISTICS}

Studies of the capacitance $(C)$ and the conductance $(G)$ associated with the depletion region can provide additional information about the concentration and characteristics of electrically active centers. For that reason we also monitored the ac properties of the device. In standard theory of singlebarrier devices the depletion layer capacitance follows ${ }^{20}$

$$
C=A \sqrt{\frac{e \varepsilon_{r} \varepsilon_{0} N_{A}}{2\left(V-V_{\mathrm{bi}}\right)}},
$$

with $N_{A}$ the acceptor concentration, $V_{\text {bi }}$ the built-in voltage, $A$ the area of the interface, $\varepsilon_{r}$ the relative permittivity, $\varepsilon_{0}$ the permittivity of vacuum, and $e$ the elementary charge. ${ }^{21} \mathrm{Be}-$ cause in these measurements a small ac voltage is superimposed on the dc bias, the ac conductance, also sometimes called the dynamic conductance, will be equal to the derivative of the DC current and therefore depends exponentially on the voltage. Both the capacitance and conductance in this simple model are independent of the ac frequency.

Interface states and deep levels can change the capacitance and conductance and can give rise to frequency dependencies as described by Nicollian and Goetzberger. ${ }^{22}$ Figure 3 clarifies this idea for the interface states. For forward biases the interface states are completely empty (that is neutral in the case of acceptor states), while for strong reverse biases they are completely full. In either case, modulating the Fermi level with the external voltage does not result in changes in the amount of charge stored on the interface states. For certain biases the Fermi level can be degenerate with the interface-state levels (middle plot in Fig. 3). Modulating it with the ac voltage results in charge flowing in and out of the interface states. This current is then proportional to the derivative of the ac voltage and is visible as an extra capacitance added to the capacitance of Eq. (1) at certain voltages. Because energy can be lost in the process of transferring the

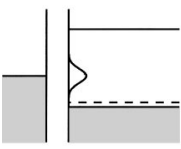

(a) forward

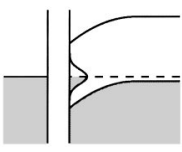

(b) no bias

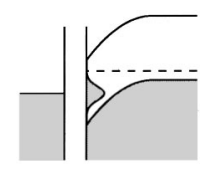

(c) reverse
FIG. 3. Schematic band diagram of an MIS diode with interface states. (a) For forward biases, the Fermi level (dashed line) lies completely below the interface states which are therefore completely empty (neutral). (b) For some biases the Fermi level is resonant with the interface-state levels. In this case, a small change in the bias voltage results in charge flowing in or out of the interface. This is visible as an increased conductance and capacitance. (c) For strong reverse biases the levels are completely occupied and there is no current upon changes in bias.

charge, some of the associated current is in phase with the ac voltage and is therefore visible in a contribution to the conductance.

For low frequencies the charges can follow the Fermi level modulation without problem. For high frequencies the trapping and detrapping times are much longer than the ac period and the interface levels cannot reach thermal equilibrium. In that case the capacitance and conductance reduce to the depletion region values mentioned at the beginning of this section. Note that there is no dc contribution to the conductance since the number of interface states is finite; the conductance drops to zero at low frequencies, whereas the capacitance levels off (since the latter is calculated by dividing the out-of-phase current by the ac frequency, $I_{90^{\circ}}$ $=\omega C-V_{\text {ac }}$, where $I_{0^{\circ}}=G-V_{\text {ac }}$ defines $\left.G\right)$. There is a maximum response in the $G-V$ plot when the (radial) modulation frequency reaches the reciprocal trap filling time, $\omega=1 / \tau$. For very slow states this can be in the range of the dc scanning speed.

For states that do not respond to the ac signal, but still respond to the bias changes complicated effects take place. This is equivalent to the case of deep, nonresponding bulk states which will be discussed in the next section. States that do not even respond to the bias changes can still influence the $C-V$ and $G-V$ plot: fixed charges can cause a constant voltage drop and a rigid shift of the $C-V$ and $G-V$ plots along the voltage axis. ${ }^{22}$

To summarize, for MIS tunnel diodes with interface states it has been predicted that there is a peak in the $C-V$ and $G-V$ plots, with an intensity depending on the ac frequency and a voltage position depending on the distribution of the interface states in the forbidden gap.

Compared to interface states, deep levels that are homogeneously distributed in space have very similar behavior but show themselves in the $C-V$ plots stepwise, up-to a certain voltage, rather than as a peak as shown above. This will be discussed later, in the section on Mott-Schottky plots.

Figure 4 presents the $G-V$ data. The freshly mounted sample shows a peak at $0.4 \mathrm{~V}$ forward bias at $1 \mathrm{kHz}$ when corrected for the exponential dc conductances. We attribute this to interface states, as previously described.

The same sample placed in vacuum for longer periods has a much less pronounced peak in $G-V$, most of the interface states have been removed or changed by the pump- 

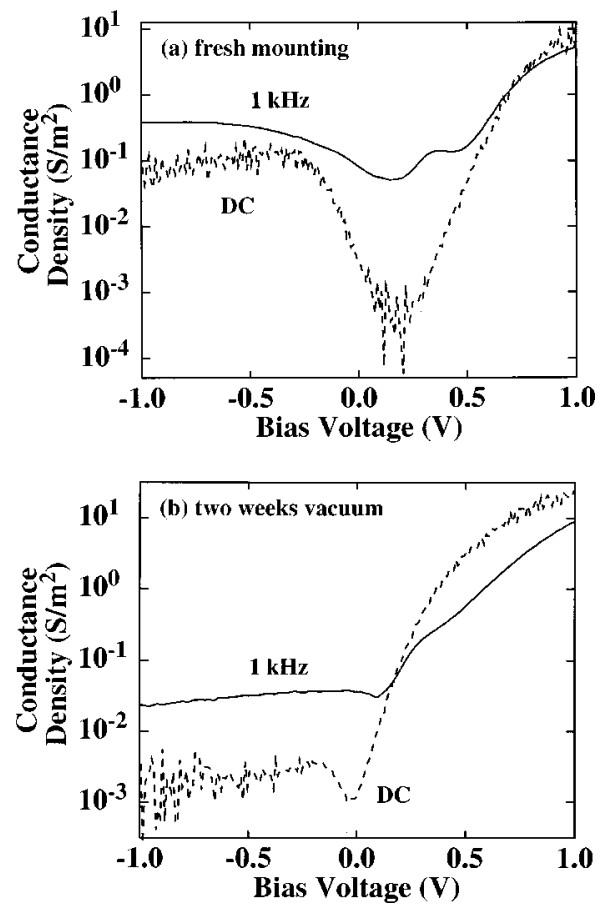

FIG. 4. (a) Conductance density (1/AR) plot at room temperature for the freshly mounted sample at $1 \mathrm{kHz}$ (solid). When the dc peak (dashed) is subtracted from it, a positive peak at $\sim 0.4 \mathrm{~V}$ remains. (b) After pumping for several weeks the peak is reduced, indicating the removal of the interface states. The dc conductance is calculated from the derivative of the $I-V$ curves of Fig. 2.

ing. This is consistent with the dc data as presented in the previous section.

Figure 5 summarizes the frequency dependence of the peak in the loss $(G / \omega)$. This was measured in another sample where the dc conductance was much lower (see inset) and the peak more pronounced. The figure shows that the largest response is for the lowest frequencies. Apparently, the maximum peak height in the $G-V$ plot would occur for frequencies well below our measurement window $(100 \mathrm{~Hz}-1$ $\mathrm{MHz}$ ), which indicates that the interface states are rather deep and the filling time is even longer than the $2 \mathrm{~ms}$ corresponding to $100 \mathrm{~Hz}$. The shift of the peak towards lower

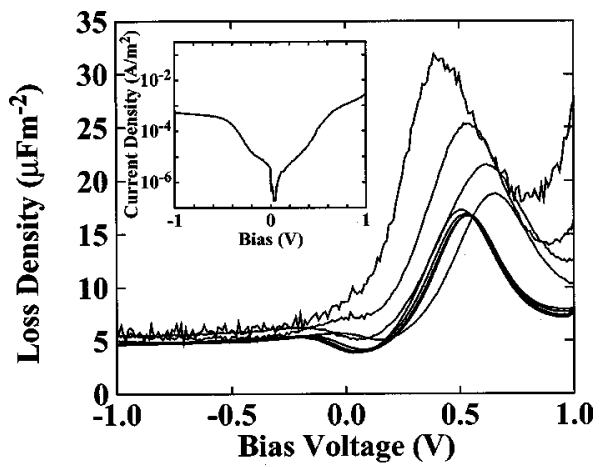

FIG. 5. Frequency dependence of the loss $(1 / \omega R)$ at room temperature for a sample with low conductivity (see inset). The low background conductance enables the observation of a peak in forward bias which is attributed to interface states. The largest response of the interface states occurs at the lowest frequency $(200 \mathrm{~Hz})$ and slowly decreases for higher frequencies $(500$ $\mathrm{Hz}, 1 \mathrm{kHz}, 2 \mathrm{kHz}$ ). After $3 \mathrm{kHz}$ it levels off. voltages for decreasing frequencies is caused by the ability to probe deeper states with longer relaxation times as the ac frequency is reduced. For higher frequencies the peak height in $G / \omega$ reaches a constant value, and a well at $0.1 \mathrm{~V}$ forward bias sets in. Both these effects, the leveling off of the peak height and a well rather than a peak, cannot be explained with the above theory. We will show what the cause is for the well.

Summarizing, the peak in $G-V$ data correlates very well with the features seen in the $I-V$ curves, and can be adequately described in the model of interface states that can be altered with persistent pumping. Apparently, the interface quality is both visible in the dc measurements as well as in the ac data.

\section{MOTT-SCHOTTKY PLOTS}

It is common to present the capacitance-voltage data in so-called Mott-Schottky (MS) plots, $1 / C^{2}$ vs voltage $(V)$. In ordinary situations, the slope in such a plot reveals the acceptor concentration $N_{A}$ because, according to Eq. (1)

$$
\frac{d}{d V} \frac{1}{C^{2}}=\frac{2}{A^{2} e \varepsilon_{r} \varepsilon_{0} N_{A}} .
$$

This is applicable to the case of a single shallow acceptor (all ionized everywhere; space charge density in the depletion zone equal to the acceptor concentration), in which case the Mott-Schottky plot consists of a straight line pointing to $V_{\mathrm{bi}}$ at the voltage axis when the concentration is uniform, or the slope represents the local acceptor concentration in case of nonuniform distributions. It is quite common to determine the acceptor concentration from the Mott-Schottky plots of single-level systems.

When there are more levels in the forbidden gap the situation becomes more complex (see Fig. 6). First consider the situation of two acceptor levels, one shallow $\left(E_{A_{1}}\right.$ with concentration $\left.N_{A_{1}}\right)$ and one deep $\left(E_{A_{2}}\right.$ with concentration $N_{A_{2}}$ ), in fact so deep that in the absence of bias it nowhere drops below the Fermi level. This implies that all of the deep acceptor levels are filled (with holes) and are therefore neutral and do not contribute to the space charge and capacitance; the slope in the Mott-Schottky plot then represents only the shallow acceptor concentration. For strong reverse biases the increased band bending can force the deep acceptor below the Fermi level at a region close to the interface. Now if the level is shallow enough so that it can respond to the ac probing signal (of the order of $1 \mathrm{kHz}$ ) it will start contributing to the capacitance. The apparent concentration is then the sum of the two concentrations of the acceptors. This is visible in a sudden drop and a reduction of the slope to $1 /\left(N_{A_{1}}+N_{A_{2}}\right)$ for biases smaller (more reverse) than $V_{\mathrm{bi}}-\left(E_{F}-E_{A_{2}}\right),{ }^{23,24}$ as is easily seen in Fig. 6. Also, note that the apparent built-in voltage seems to decrease; the intersect of the slope with the voltage axis is lowered to $V_{\mathrm{bi}}$ $-N_{A_{2}}\left(E_{F}-E_{A_{2}}\right) /\left(N_{A_{1}}+N_{A_{2}}\right)$.

If the level is too deep and the probing frequency is too high compared to the characteristic level filling and emptying times $\tau$, it does not respond anymore. It is still visible in 


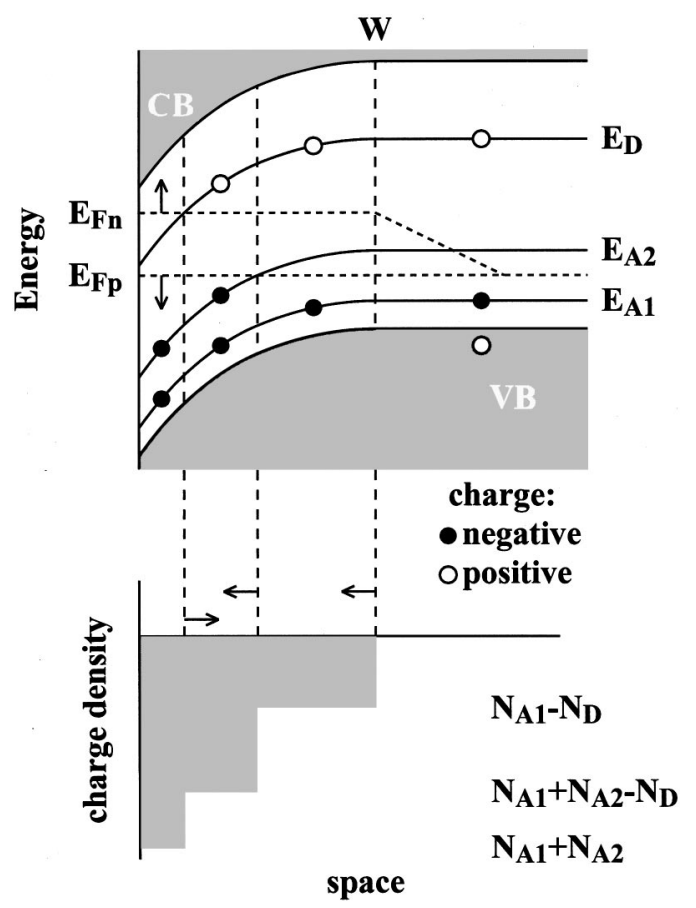

FIG. 6. Schematic energy diagram for a system with two acceptor levels and one donor level at forward bias. At a certain place the band bending is large enough to force the deep acceptor level below the (hole) Fermi level and it starts contributing to the space charge and capacitance. Closer to the interface the (electron) Fermi level crosses a donor level which now stops to compensate the acceptors and increases the space charge density. The arrows indicate the movement of the boundary edges upon an increase of the (forward) bias.

a reduction in the slope in the Mott-Schottky plot, but now without the sudden drop. ${ }^{23}$ It is important to note that the slope is no longer linear and a straight-line fitting does not reveal the true acceptor concentration anymore; Eq. (2) is no longer valid. Also, any extrapolation of the local slope to $1 /$ $C^{2}=0$ increases to beyond $V_{\mathrm{bi}}$.

If the second level is not a majority trap (in this case acceptor level), but instead a minority trap, the situation is again a little different. Instead of the majority (quasi) Fermi level, $E_{F p}$, as above, we now have to consider the minority (quasi) Fermi level, $E_{F_{n}}$. These two can substantially deviate from each other; large numbers of electrons injected into the conduction band move $E_{F_{n}}$ up. The difference between the two at the interface is actually equal to the bias. Whereas the Fermi level for holes is flat throughout the entire polymer, the Fermi level for electrons is flat in the depletion zone and from there linearly falls back to meet $E_{F_{p}}=E_{F}$ somewhere in the bulk of the polymer. ${ }^{15}$ The exact movement of this Fermi level and its slope in the bulk, and hence the intersect with the donor level, depend on parameters such as the injection ratio, the mobility of the electrons and the electron-hole recombination rate. Therefore, it is not easy to calculate the precise effects of a minority level on the capacitance. When the donor level is completely above the (minority) Fermi level everywhere, it is completely ionized and acts as compensation for the acceptors. The slope we see in the MottSchottky plot is then proportional to $1 /\left(N_{A}-N_{D}\right)$. This is especially the case for shallow donors. When the donor level

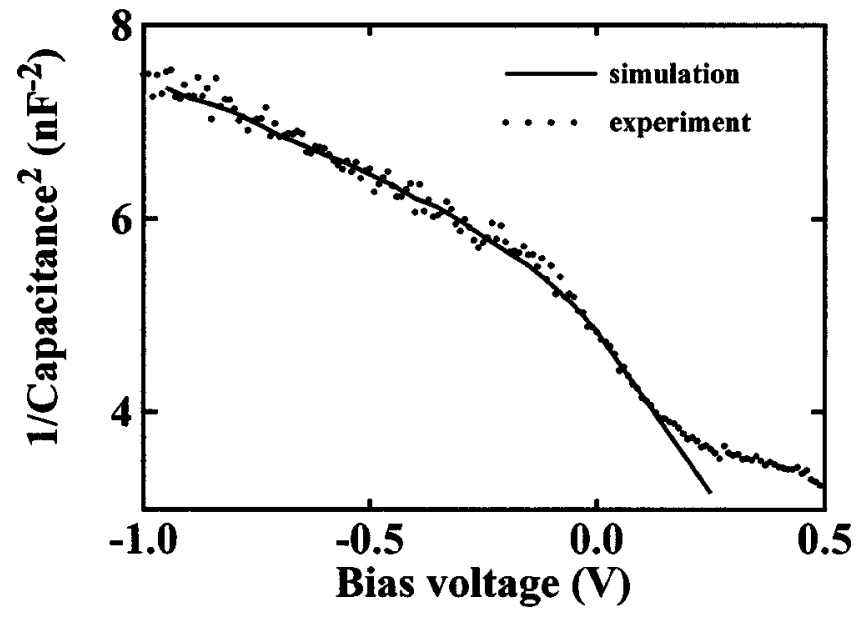

FIG. 7. Typical Mott-Schottky plot $\left(1 / C^{2}\right.$ vs $\left.V\right)$ measured at $100 \mathrm{~Hz}$ at room temperature (modulation depth: $50 \mathrm{mV}$, voltage step: $20 \mathrm{mV}$ per second). The dots show the experimetal data, while the solid line represents a simulation with two acceptor levels. The shallow one is responding to the ac signal and has a concentration of $4.3 \times 10^{15} \mathrm{~cm}^{-3}$, while the deep one $(0.75$ $\mathrm{eV}$ above the Fermi level) is not responding and has a concentration of $6.7 \times 10^{16} \mathrm{~cm}^{-3}$. An $\epsilon_{r}=5$ was assumed.

drops below the Fermi level somewhere, the effects become most complex. However, it is important to note that, as for all levels that are homogeneously distributed in space, the effects are visible stepwise in the voltage in contrast to certain effects that have a voltage window.

Finally, for normal temperatures, the Fermi-Dirac distributions over the levels make the effects smooth out a little. This is further enhanced by the use of finite ac amplitudes, in our case $50 \mathrm{mV}$. A sudden step in the capacitance, for instance, is in reality visible as a more gradual step. Also, interface states as described in the previous section can add structure to the Mott-Schottky plots.

With this information we can analyze the $C-V$ data of the MEH-PPV. Figure 7 shows a typical Mott-Schottky (MS) plot taken at an ac frequency of $100 \mathrm{~Hz}$. It shows a linear slope at reverse bias changing to a steeper slope at forward bias. Such plots with a sudden change in slope are often encountered in semiconducting polymers, see for example Refs. 25 and 26, and can be described well with the above theory. In the same figure, a simulation is shown including two levels, a shallow level with a concentration of $4.3 \times 10^{15} \mathrm{~cm}^{-3}$ and a deep (nonresponding) level $0.75 \mathrm{eV}$ above the Fermi level with a concentration of $6.7 \times 10^{16}$ $\mathrm{cm}^{-3}$. The extrapolation of the forward bias slope to the voltage axis reveals a built-in voltage of $0.75 \mathrm{~V}$. This value is in agreement with the predicted value on the basis of the band-structure data (see Sec. II). The idea might occur to the reader that the change in slope in the MS plots instead is due to a spatial change in impurity concentration. In this case, the reverse-bias slope would indicate a sudden increase of the acceptor concentration from $4.3 \times 10^{15}$ to $1.3 \times 10^{16} \mathrm{~cm}^{-3}$ as we move away from the interface. It is, however, unlikely that for different devices with different impurity concentrations the change in slope should always occur at the same voltage, as it does. Different sample preparations resulting in different impurity densities would cause a wide range of the 


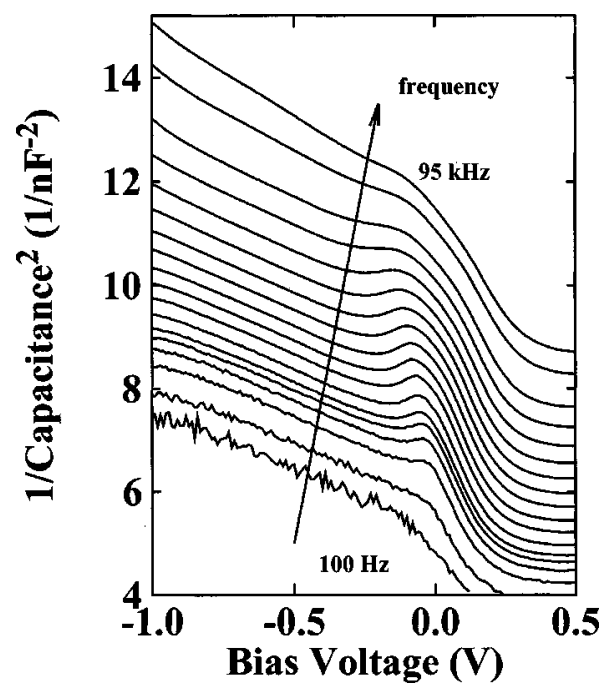

FIG. 8. Frequency dependence of the Mott-Schottky plots such as shown in Fig. 7 at room temperature. A peak appears at small biases for intermediate frequencies.

sizes of the depletion width (at the same voltage) and hence a wide range of voltages where the edge of the depletion zone hits the boundary of the higher-doped region. On the other hand, in the above theory, the position of the change in slope is determined by the energetic positions of the levels in the gap, something that is expected to be independent of doping details.

For the freshly mounted sample, a much reduced slope was found compared to the pumped sample. This can be caused by a higher acceptor concentration, or, alternatively, by an increased absorption of the voltage drop by the interface layer. Again this would hint at an alteration of the interface by the persistent pumping.

In Fig. 8 the frequency dependence of the MS plots is shown. At frequencies above $200 \mathrm{~Hz}$ a structure appears at the small-bias range which again disappears at higher frequencies. This anomalous structure cannot be explained by the above theory of deep levels; a responding level could cause a positive slope in the MS plots, but would obviously also be visible at the lowest frequencies. Moreover, in a simulation the reverse-bias branch would drop far below the experimental data. On the other hand, a nonresponding deep level does not show itself as a peak in the MS plots (but is visible as a reduction in slope up to a certain voltage), nor does it have peaked response in frequency. In conclusion, the incorporation of deep levels into the model seems to predict the low-frequency data, but fails to explain the anomaly in the region of small bias for intermediate frequencies.

Similar anomalous structures in Mott-Schottky (MS) plots have often been reported in the literature (for instance for heterojunctions such as $\mathrm{CuInSe}_{2} / \mathrm{CdS}^{27}$ ), and are there explained in terms of (a) back-rectifying contacts, (b) instrumental artifacts, (c) deep levels, ${ }^{28}$ (d) inductive effects due to minority carriers. ${ }^{18}$ In principle, a rectifying back contact could produce a local minimum in the $C-V$ data by combining the voltage dependencies of the capacitances of the two Schottky-barrier interfaces. Since gold, our back contact, makes a good ohmic contact with MEH-PPV we do not ex- pect any rectifying properties. Instrumental artifacts such as parasitic capacitance of the cables were carefully checked and none were found below $1 \mathrm{MHz}$. Such effects normally start dominating at high frequencies while the devices studied still displayed normal capacitance in the high-frequencymeasurement range. As described above, deep levels cannot explain the anomalous effect. The structure might be due to interface states, but then it would rather be expected to be most pronounced at the lowest frequencies, in contrast to the observed frequency dependence. We present an alternative explanation, namely the inductive effects of minority-carrier injection. $^{18,29}$

The underlying physics have been well described by Misawa for $p n$ junctions: ${ }^{29}$ In the bulk region of the semiconductor the minority-carrier current is mostly caused by diffusion, whereas the majority-carrier current is caused by drift. They move under the influence of the electric field caused by the IR voltage drop in the bulk. Diffusion is a rather slow process and the current cannot follow the voltage changes instantaneously; the current starts lagging behind the voltage and a shift of the phase of the current relative to the voltage occurs. If the phase shift is large this effect can imitate negative capacitance (equivalent to inductance) and negative resistance; in the measurements one can observe a reduced capacitance and increased conductance. It must be noted that this is not a true capacitance in the sense that the device can store charge (this is the definition of static capacitance), but is rather an as-measured capacitance caused by a phase shift of the ac current.

Green and Shewchun present the same idea of the minority-carrier effects upon the ac characteristics of the device. They show how Schottky diodes can become inductive under moderate bias due to the presence of small concentrations of minority carriers. ${ }^{18}$ They also note that, by increasing the frequency of the applied signal, the corresponding minority-carrier disturbance can be readily attenuated and the capacitance curves approach those expected on the basis of a one-carrier model. Furthermore, they were able to model the effects with the help of equivalent circuits containing frequency- and voltage-dependent components. These equivalent circuits do not reveal so much of the underlying physics, though.

According to Misawa, the total impedance of the $p$-type semiconductor can be described by the sum of the three terms

$$
Z=Z_{p_{1}}+Z_{p_{2}}+Z_{p_{3}} .
$$

The first term is purely resistive and is what is expected from the normal conductivity of the interface, namely the depletion width divided by the conductivity of the material caused by majority carriers:

$$
Z_{p_{1}}=\frac{W}{e \mu_{p} p_{p}} .
$$

The magnitude of the second term is proportional to the diffusion length of minority carriers divided by the majoritycarrier conductivity $L_{n} / e \mu_{p} p_{p}$ which is multiplied by the fraction of the minority-carrier AC current of the total ac current at the interface, $I_{n} / I$. This is further multiplied by a 


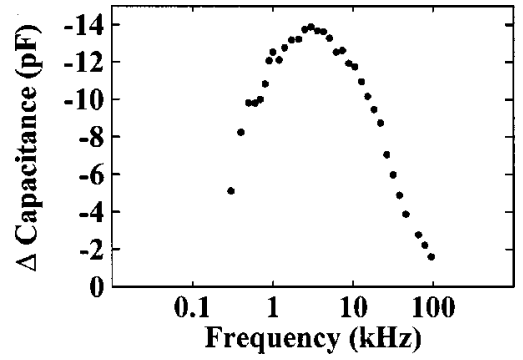

FIG. 9. Frequency dependence of the amplitude of the well in the $C-V$ plots, extracted from plots such as shown in Fig. 8. The maximum at $3 \mathrm{kHz}$ indicates a minority carrier lifetime of $100 \mu \mathrm{s}$.

factor $\Phi$ containing the phase which depends on the frequency $(\omega)$ and minority-carrier lifetime $\left(\tau_{n}\right)$ and is $180^{\circ}$ $(-R)$ for $\omega$ approaching zero and turns to $90^{\circ}(L$, or $-C$ ) for higher frequencies. In a phase diagram $\Phi$ is always in the quadrant $(-R, L)$ and there is a maximum inductance for $\omega \tau_{n} \approx 2$ :

$$
Z_{p_{2}}=\frac{L_{n}}{e \mu_{p} p_{p}} \frac{I_{n}}{I} \frac{-1}{\sqrt{1+i \omega \tau_{n}}} .
$$

The last term, $Z_{p_{3}}$ is more complex; for instance it also depends on the ratio of injected-minority-carrier density to thermal-equilibrium-majority-carrier density, $n / p_{p}$. It also has a more complicated phase factor, which can make the impedance lie in any quadrant in a phase diagram:

$Z_{p_{3}}=\frac{L_{n}}{e \mu_{p} p_{p}} \frac{n}{p_{p}}\left(\frac{I_{n}^{\mathrm{dc}}}{I} \frac{1}{1+\sqrt{1+i \omega \tau_{n}}}-\frac{I^{\mathrm{dc}}}{I} \frac{1}{\sqrt{1+i \omega \tau_{n}}}\right)$,

with $I_{n}^{\mathrm{dc}}$ the minority-carrier dc current and $I^{\mathrm{dc}}$ the total dc current.

As is clear from Fig. 8, in our device the minority-carrier current must be a substantial fraction of the total current for small biases; making $Z_{p_{2}}$ and/or $Z_{p_{3}}$ large and disappearing again at larger forward biases. This explains the peaked behavior in the Mott-Schottky plots. Figure 9 shows the frequency dependence of the amplitude of the well in capacitance for a different sample. The maximum in inductance occurs at around $3 \mathrm{kHz}$ indicating a minority-carrier lifetime of $100 \mu \mathrm{s}$.

An accompanying resistance plot is shown in Fig. 10. In this plot, an exponential conductance has been fitted and sub-

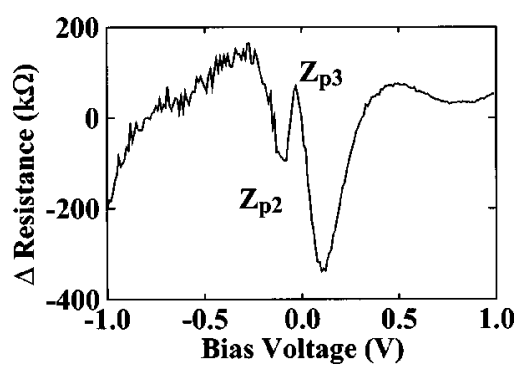

FIG. 10. At the same voltage where the structure appears in the MottSchottky plots (see Fig. 8), there is a wide well $\left(Z_{p_{2}}\right)$ and a narrow peak $\left(Z_{p_{3}}\right)$ in the ac resistance. To obtain the plot an exponential conductance was subtracted from the data; measured at $1.2 \mathrm{kHz}$.

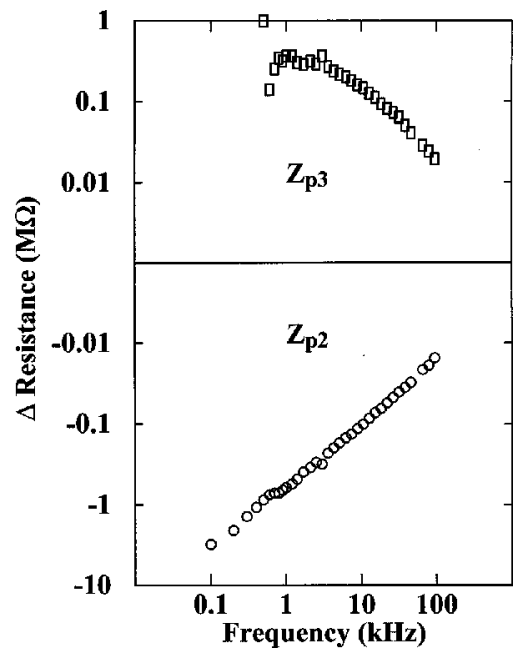

FIG. 11. Frequency dependence of the well $\left(Z_{p_{2}}\right.$, bottom) in the acresistance plots (see Fig. 10) is linear in this range, while the frequency dependence of the peak ( $Z_{p_{3}}$, top) is more complex. This follows the theory of Misawa (see Ref. 29) for minority-carrier effects.

tracted from the data. It shows a structure of a broad well, stretching about $0.5 \mathrm{~V}$, and inside it a positive-resistance narrow peak of $0.1 \mathrm{~V}$ width. On the basis of the frequency dependence of the amplitudes (see Fig. 11), we can assign the broad well to $Z_{p_{2}}$ and the narrow peak to $Z_{p_{3}}$. Apparently there are voltage regimes that favor the $Z_{p_{2}}$ term (always negative resistance) and regimes that favor the $Z_{p_{3}}$ term. This can happen because $Z_{p_{3}}$ depends, apart from the relative minority-carrier current, like $Z_{p_{2}}$, also on the relative minority-carrier concentration. As shown above, $Z_{p 1}$ and $Z_{p 2}$ two terms can have different frequency dependence. We conclude that the relatively large minority-carrier effects at small biases are responsible for the capacitance and conductance anomalies presented here.

One final thing to note is that although the above theory gives an adequate explanation for the effects seen in our samples, we cannot completely rule out the possibility that they are related to the silicon surface and not to the intrinsic properties of the polymer. Gold-silicon junctions, made as reference samples, sometimes show effects in the $C-V$ plots that resemble the ones reported here.

\section{ADMITTANCE SPECTROSCOPY}

In reality the device we are measuring does not only consist of the interfacial layer (depletion layer), but is part of a larger structure which also contains the bulk material. In most cases this is a nuisance - the bulk resistance and capacitance can make the measured values deviate substantially from the interface values-but in some cases we can make use of this effect. Figure 12 shows the equivalent circuit used to represent the total device. A small calculation can show that even when all components in the circuit are frequency independent, the measured capacitance and resistance of the total circuit depend on the probing frequency. Assuming that the bulk resistance and capacitance $\left(R_{b}\right.$ and $\left.C_{b}\right)$ are much smaller than their depletion layer siblings $\left(R_{d}\right.$ 


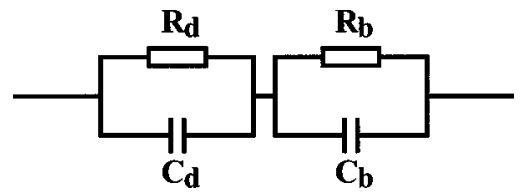

FIG. 12. Equivalent circuit used to model the admittance spectroscopy data. It consists of a loop representing the bulk (denoted by the subscript " $b$ ") in series with a loop representing the depletion layer (subscript " $d$ ").

and $C_{d}$ ), the measured capacitance and resistance are equal to the depletion layer values at low frequencies, while for high frequencies they are equal to the bulk values. Somewhere between is a turning frequency. This frequency is easily found when the data are plotted in the form of a loss tangent $(\tan \delta=1 / \omega R C)$. This plot will have a local maximum at a radial frequency: ${ }^{30}$

$$
\omega_{\max }=\frac{1}{R_{b} \sqrt{C_{b}\left(C_{b}+C_{d}\right)}} .
$$

Figure 13 shows an example of the loss tangent (measured at a temperature of $200 \mathrm{~K}$ ) with a maximum around $60 \mathrm{kHz}$. Because the temperature dependencies of the capacitances are relatively insignificant, the position of the peak should have the same temperature dependence as the bulk resistance. Furthermore, when we assume that the bulk resistance is governed only by the activation energy (constant mobility and constant density of states in the valence band), the resistance will follow

$$
R_{b} \propto \exp \left(-E_{a} / k T\right),
$$

with $E_{a}$ the bulk activation energy. Figure 14 shows the result. The Arrhenius plot of the position of the peak in the loss tangent of Fig. 13 versus "coldness" (reciprocal temperature) shows a straight line, revealing an activation energy of $0.12 \mathrm{eV}$.

The frequency at the maximum of the loss tangent has another important aspect. For all the ac measurements, we have to take care to always use frequencies below this turning point in order to make sure that we are measuring the depletion layer properties. This is just an electronics effect and, apart from the trait described above, there is not much physics in it. For the classic semiconductors it is never a big

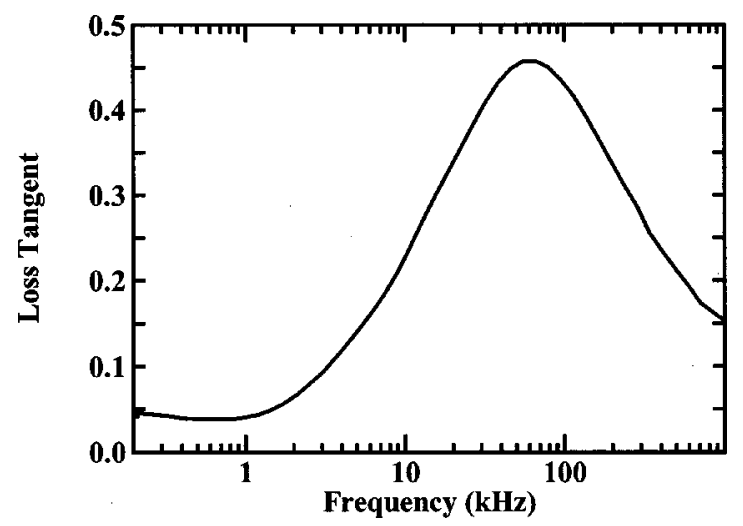

FIG. 13. Example of a spectrum of the loss tangent $(1 / \omega R C)$ at $200 \mathrm{~K}$. A maximum occurs at $60 \mathrm{kHz}$.

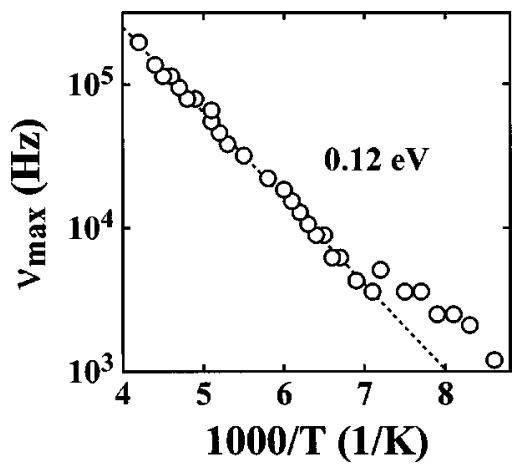

FIG. 14. Arrhenius plot of the frequency position of the maximum in the loss tangent of Fig. 13 as a function of reciprocal temperature, revealing an activation energy $E_{A}=0.12 \mathrm{eV}$.

issue; normally for these materials the turning frequency is very high and we are always well below it. For polymers, especially those with high bulk resistivity, it can become a problem, though, and care has to be taken to choose sufficiently low frequencies in the measurements. The tradeoff of choosing low frequencies is that the noise increases rapidly (so-called $1 / f$ noise) and this sets the lower limit of usable frequencies. For the samples under study, the cutoff frequency lies well above $100 \mathrm{kHz}$ at room temperature and all the data represent the depletion region.

\section{TEMPERATURE STIMULATED CURRENT}

In a thermally stimulated current (TSC) experiment, the device is cooled down under strong forward bias. When the lowest temperature is reached the bias is removed and the sample is slowly warmed up while monitoring the current. In forward bias, the bands are flat and the deep acceptor level is nowhere below the Fermi level; they are all neutral. When the bias is removed the band bending is restored, but the temperature is too low to allow for emission of holes from the deep levels. When the temperature is slowly increased the holes will be reemitted at a certain temperature. The electric field within the depletion layer will drift them towards the polymer-side electrode (away from the interface) and a negative (reverse) current results. This is visible in the temperature stimulated current (TSC) of Fig. 15. When all the

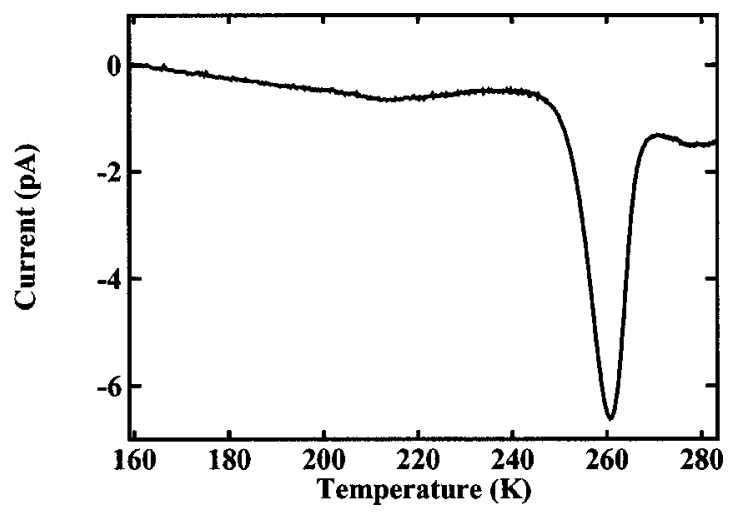

FIG. 15. Temperature stimulated current at $0 \mathrm{~V}$ bias (cooled down at +1.0 V). The integration of the negative peak indicates a level with a concentration of $4 \times 10^{16} \mathrm{~cm}^{-3}$. 
holes have been emitted and the device has reached thermal equilibrium again, the current drops back to zero.

The number of holes emitted from the acceptors and contributing to the TSC can be found by integrating the peak: $-6.7 \mathrm{nC}\left(4.2 \times 10^{10}\right.$ defects. $)$ The depletion volume under the circular electrode is about $9 \times 10^{-13} \mathrm{~m}^{-3}$ at $0 \mathrm{~V}$ bias (based on a $300 \mathrm{~nm}$ depletion width and an electrode diameter of $2 \mathrm{~mm}$ ) and 0 at strong forward bias. This gives an estimate for the concentration of the deep acceptor responsible for the peak in Fig. 15; $N_{A}=4 \times 10^{16} \mathrm{~cm}^{-3}$. Whether this is correct, or the peak in TSC is due to interface states is not clear. The integrated current of $-6.7 \mathrm{nC}$ would correspond to an interface-state density of $1.3 \times 10^{12} \mathrm{~cm}^{-2}$, a reasonable number (for silicon surfaces ${ }^{15}$ ).

The same results can be obtained from a voltaic current transient. When the bias is suddenly removed, a small current lingers on. This is the current that is related to the emptying (or filling) of deep levels which can take a long time to reach thermal equilibrium. The integrated current is also here equal to the number of deep impurities in the region that has fallen outside (or inside) the new depletion width. Figure 16 shows an example. Here the voltage was changed from -1 to $0 \mathrm{~V}$ and the depletion width will have shrunk by about 75 $\mathrm{nm}$. Hence, the integrated current of $2.5 \mathrm{nC}$ indicates an acceptor density of $\sim N_{A}=6 \times 10^{16} \mathrm{~cm}^{-3}$. At the moment we have no clear proof that these currents stem from bulk states or from inside the polymer. Future experiments will address this problem with the use of more advanced TSC and current-transient techniques.

Returning to the TSC measurements, the depth of the level can, in principle, be estimated when the position of the peak in TSC is determined as a function of the heating rate. For this to be accurate, the temperature scanning rate has to be very constant and well known over a long period of time. A much more direct and precise way to determine the level depth is via the temperature dependence of the loss tangent, as previously described, for the shallow level, or transient spectroscopy for the deeper levels. This will be described in Sec. IX.

\section{CAPACITANCE TRANSIENT SPECTROSCOPY}

In this technique we observe the transient of the capacitance ( $C$ vs $t$ ) after a bias change. Levels are allowed to reach thermal equilibrium at a certain voltage. The transient is then recorded after switching the bias to a lower voltage. The charges that were originally trapped are emitted with a characteristic time, $\tau$. The activation energy of the trap is calculated by noting that the emission rate, $e_{n}=1 / \tau$, of the carriers from a trap of depth $E_{a}$ at temperature $T$ is given by $^{31}$

$$
e_{n}=1 / \tau \propto T^{2} \exp \left(E_{a} / k T\right),
$$

where $k$ is the Boltzmann constant. Measuring the time constant of the transients as a function of the temperature will then yield the trap depth, $E_{a}$.

The capacitance usually shows an immediate drop when the voltage is lowered, caused by the fast growth of the depletion region. The speed at which this happens is only

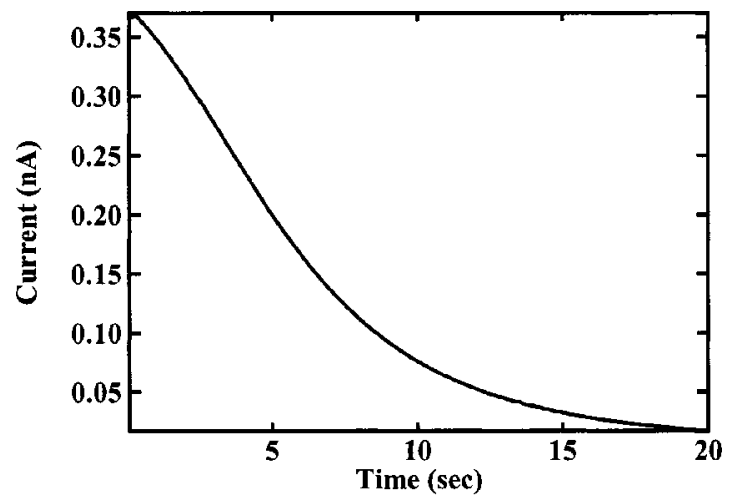

FIG. 16. Voltaic current transient obtained after switching the bias from -1 to $0 \mathrm{~V}$. The integrated current $(2.5 \mathrm{nC})$ corresponds to an acceptor density of approximately $N_{A}=6 \times 10^{16} \mathrm{~cm}^{-3}$.

limited by the mobility of the majority carriers and is normally too fast for the observation of a transient. This is then followed by a long-lived signal. For a majority-carrier trap we expect a negative capacitance transient (upward trend); charges are released from the acceptors and the depletion width decreases, observable as a slowly increasing capacitance over time. For minority-carrier traps-traps that communicate more readily with the minority-carrier band rather than with the majority-carrier band-the transients are expected to be positive (downward trend) $;^{31}$ upon a lowering of the bias the electron-Fermi level moves down and the electrons are released from the traps resulting in a decrease of the space charge density and hence an increase in the depletion width and a reduced capacitance. It is obvious that for a minority-carrier transient to be observed, minority carriers have be able to be injected into the polymer. To make sure of this, we made use of the heterojunction of heavily doped $n$-type silicon and the $p$-type polymer.

In standard deep-level-transient-spectroscopy (DLTS) methods the device is primed many times per second. This is possible because for the classic materials such as silicon the decay times of the transients lie well below $1 \mathrm{~s}$ for all temperatures. The procedure is to take many transients per second and average them. To reduce the amount of calculation required, from these data only two data points at a fixed moment in time are kept (the so-called time window). Recently, in Laplace DLTS, all data are taken into the calculation (made possible by the availability of cheap datamanipulating power in modern computers), and a larger sensitivity and resolution is obtained. For the polymers, the transients are much slower. Each transient is in the order of $100 \mathrm{~s}$. For such long scans, averaging is not possible unless the temperature stability is compromised. This also inhibits the use of time windows; the scarce amount of data necessitates the full use of the data. We fitted a limited number of exponentials, up to three, to all the data points in a scan. In the few cases where the number of exponentials to use in the fit was not evident from the scan or the fit quality was bad the data were rejected.

Figure 17 shows that, in our case, by an appropriate pulse scheme, it is possible to prime either the minority- or the majority-carrier trap levels. The majority-carrier traps 


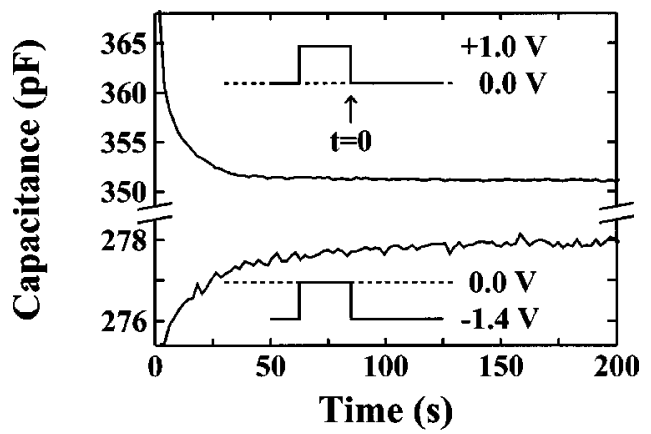

FIG. 17. By a suitable choice of the pulse we can make the transients reveal a majority trap in a 0 to $-1.4 \mathrm{~V}$ pulse or a minority trap in a forward injecting pulse +1 to $-0.4 \mathrm{~V}$. Measured at room temperature.

can be detected using a filling pulse of zero bias, and minority-carrier traps are revealed by injecting electrons into the polymer in a forward-bias pulse of $1.0 \mathrm{~V}$. A complication is that forward-injecting bias pulses, because of the collapsing of the depletion region, can also significantly perturb the occupation of interface states (which are more homogeneously distributed in energy and hence give nonexponential contributions to the capacitance transients), which is not desirable because our interest is to prime only discreet bulk traps. Therefore, during the experiments the sample was kept under zero bias and then subjected to a reverse step of -0.8 $\mathrm{V}$. The corresponding transient capacitance under reverse bias was recorded at a series of stabilized temperatures to provide the time constants $(\tau)$.

Although we did not forward bias the pn junction, we observe both types of transient corresponding to majorityand minority-type behavior. This reversal in the sign of capacitance change is illustrated in Fig. 18, where for a certain temperature both types of behavior are visible at the same time. The observation of minority-type transients suggests that electron diffusion from the $n^{+}$silicon is enough to fill minority traps in the polymer. This is consistent with the results of the ac measurements where we showed evidence for minority-carrier currents in the small-bias range.

Figure 19 summarizes the decay times found in the transients as a function of temperature. The solid and open circles represent majority and minority traps, respectively. Equation (9) was then fitted to these data points. There is

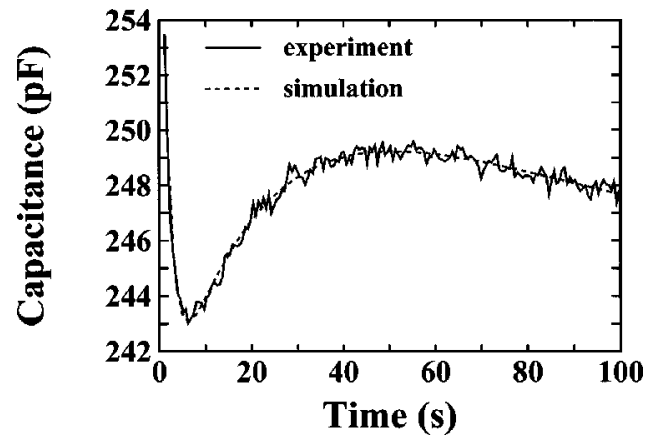

FIG. 18. At $-5^{\circ} \mathrm{C}$, three traps are visible in the same scan. A tripleexponential curve was fitted to it (dashed line). Measured at $1 \mathrm{kHz}, 100 \mathrm{mV}$ ac level, after a voltage step from 0 to $-0.8 \mathrm{~V}$.

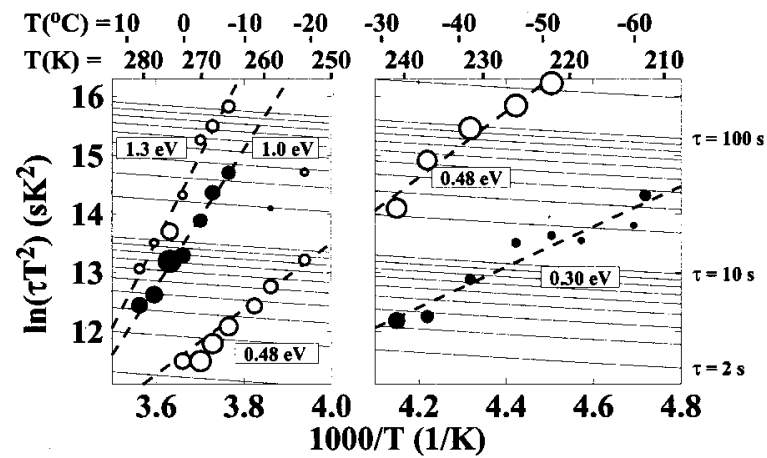

FIG. 19. $T^{2}$-corrected decay times $\tau$ as a function of coldness (reciprocal temperature) for two runs. Solid circles denote majority-type transients, while open circles indicate minority-type trap levels. The area of each point is proportional to the transient amplitude ( $\times 5$ in right plot). From these plots four trap level depths can be determined according to Eq. (9). They are summarized in Table I and Fig. 20.

clear evidence for at least four trap levels, two minority ones (activation energies: 0.48 and $1.3 \mathrm{eV}$ ) and two majority ones (activation energies: 1.0 and $0.3 \mathrm{eV}$ ). The data points related to these traps are so closely spaced that the exact determination of the time constants is cumbersome and depends, for instance, on the fitting procedure. Hence, the activation energy of the trap levels have a wide margin of error (about $20 \%$ ). It has to be noted however that there is no doubt of the existence of the above mentioned levels. Figure 20 and Table I summarize all the levels found in this work.

For another trap, one that is visible at room temperature and is not presented in Fig. 19, the temperature is stable enough to do a series of transients with different pulse lengths. The procedure is to have the device in reverse bias and remove the bias for a certain time $\tau$, the pulse length. The amplitude of the transient as a function of the pulse length can reveal information about the type of the defect responsible for the transient. ${ }^{32,33}$ For defects that can capture

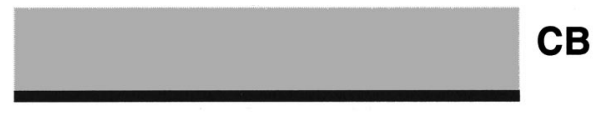
DF2

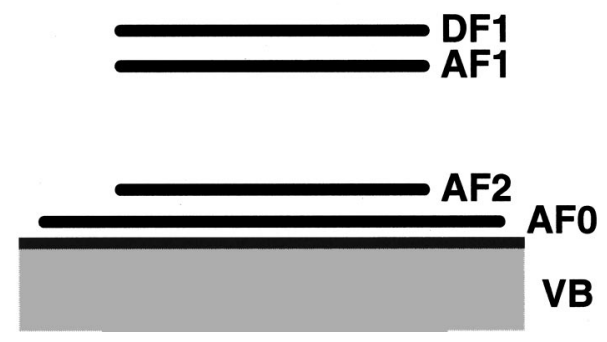

FIG. 20. Graphical presentation of the levels found in this work placed in the forbidden gap of the MEH-PPV. The shallowest acceptor level, AF0, was found via the loss-tangent data (see the section on admittance spectroscopy), while the others were found via capacitance-transient spectroscopy; see Table I for the numerical details. 
TABLE I. Trap levels detected by transient spectroscopy and admittance spectroscopy. See Fig. 20 for a graphical presentation of these levels.

\begin{tabular}{ccc}
\hline \hline Label & Type & Energy $(\mathrm{eV})$ \\
\hline AF0 & acceptor & 0.12 \\
AF1 & majority & 1.0 \\
DF1 & minority & 1.3 \\
AF2 & majority & 0.30 \\
DF2 & minority & 0.45 \\
\hline \hline
\end{tabular}

and emit single charges, the amplitude of the transient $\Delta C$ follows:

$$
\Delta C(\tau)=\Delta C_{\infty}[1-\exp (-\lambda \tau)],
$$

with $\tau$ the pulse length and $\lambda$ a constant depending on the trap-filling time. This is the case for independent single donors or acceptors, when each trapping event is independent and has a certain fixed probability to occur. A plot of $\log \left[\Delta C(\tau)-\Delta C_{\infty}\right]$ vs $\tau$ will be a straight line. For traps that can contain more than one charge, such as extended defects, or when the density of defects becomes so high that they feel each others Coulombic field, the probability of trapping a charge per unit time depends on the number already present; the trap filling time becomes increasingly longer. The above mentioned plot will no longer be a straight line because $\lambda$ is not constant. Instead, the response is initially fast and becomes slower and slower over time; the curve bends upwards. In Fig. 21 the capacitance amplitude is plotted as a function of filling time $\tau$. The conclusion is that the transient is caused by isolated defects.

\section{SUMMARY}

We have shown how we can get important information about the MEH-PPV from current and capacitance measurements, admittance spectroscopy, and transient spectroscopy. It has been shown how the ambient conditions can change the device from minority- to majority-carrier type. This is of importance for optical applications, since large minoritycarrier injections greatly enhance the radiative efficiency of the device.

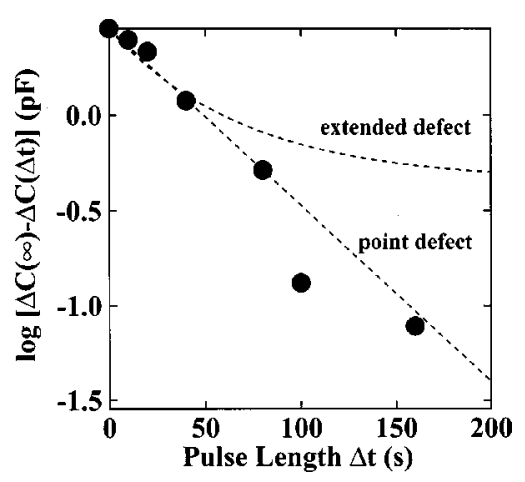

FIG. 21. Pulse-length dependence of a majority-type transient at room temperature (not visible in Fig. 19). The straight line indicates point defects rather than extended defects as the source of the transient.
Furthermore, we have shown evidence for interface states in the conductance data (see Figs. 4 and 5). These interface states seem to be alterable by persistent vacuum conditions.

Due to the combined effects of large minority-carrier currents at low voltages and the longevity of these electrons we see a pronounced structure in the $C-V$ and $G-V$ plots at small biases caused by a lagging behind of the minoritycarrier current relative to the ac voltage as was already predicted some decades ago for the classical semiconductors.

The capacitance transients were studied as a function of temperature and revealed four traps, two minority type traps and two majority type traps on top of the shallow level found by admittance spectroscopy and confirmed by the temperature dependence of the dc current. The discrete nature of the levels indicate that they belong to bulk states, rather than interface states. For the latter we expect a continuous distribution of the levels in energy and this would be visible as nonexponential decay in the transients. In our case the choice of cathode-silicon, with a clean chemically inert surface probably caused a low amount of interface states on the polymer side, enabling the observation of true bulk states.

To determine the concentrations of the impurities, we made use of Mott-Schottky plots and the results were roughly confirmed in the TSC and voltaic-current-transient data.

We have shown how the theories and measurement techniques that were originally designed for the classical semiconductors, such as $\mathrm{Si}$ and GaAs, can be applied to the new semiconductors of organic materials, such as MEH-PPV. Most of these materials have large band gaps and hence the possibility of very deep states. The relaxation times-the carrier emission and capture times from these deep states can be of the order of seconds up to hours. This places some measurement techniques, especially all the transient techniques, in a different time regime compared to classical semiconductors, although the underlying physics are the same.

\section{ACKNOWLEDGMENTS}

This work was supported by a TMR Grant, Contract No. FMRX-CT96-0083 (DG 12 - BIUO). We would like to thank D. M. Taylor for valuable discussions and K. Petritsch for measuring the film thickness.

${ }^{1}$ R. H. Friend, R. W. Gymer, A. B. Holmes, J. H. Burroughes, R. N. Marks, C. Taliani, D. D. C. Bradley, D. A. Dos Santos, J. L. Brédas, M. Lögdlund, and W. R. Salaneck, Nature (London) 397, 121 (1999).

${ }^{2}$ D. G. Lidzey, M. A. Pate, M. S. Weaver, T. A. Fisher, and D. D. C. Bradley, Synth. Met. 82, 141 (1996).

${ }^{3}$ G. Yu, Synth. Met. 80, 143 (1996).

${ }^{4}$ C. Zhang, H. von Seggern, K. Pakbaz, B. Kraabel, H. W. Schmidt, and A. J. Heeger, Synth. Met. 62, 35 (1994).

${ }^{5}$ Y. Fukada, T. Watanabe, T. Wakimoto, S. Miyaguchi, and M. Tsuchida, Synth. Met. (submitted).

${ }^{6}$ G. Yu, G. Srdanov, J. Wang, and A. J. Heeger, Synth. Met. (submitted).

${ }^{7}$ P. W. M. Blom, M. J. M. de Jong, and M. G. Munster, Phys. Rev. B 55, 656 (1997).

${ }^{8}$ P. W. M. Blom, M. J. M. de Jong, and S. Breeddijk, Appl. Phys. Lett. 71, 930 (1997).

${ }^{9}$ J. Birgerson, M. Fahlman, P. Bröms, and W. R. Salaneck, Synth. Met. 80, 125 (1996). 
${ }^{10}$ T. Osada, Th. Kugler, P. Bröms, and W. R. Salaneck, Synth. Met. 96, 77 (1998).

${ }^{11}$ I. D. Parker and H. H. Kim, Appl. Phys. Lett. 64, 1774 (1994).

${ }^{12}$ G. W. Jones, D. M. Taylor, and H. L. Gomes, Synth. Met. 85, 1341 (1997).

${ }^{13}$ A. J. Campbell, D. D. C. Bradley, E. Werner, and W. Brütting, Synth. Met. (submitted).

${ }^{14}$ F. Caciali, R. H. Friend, N. Haylett, R. Daik, W. J. Feast, D. A. dos Santos, and J. L. Brédas, Appl. Phys. Lett. 69, 3794 (1996).

${ }^{15}$ S. M. Sze, in Physics of Semiconductor Devices, 2nd ed. (Wiley, New York, 1981).

${ }^{16}$ H. C. Card and E. H. Rhoderick, Solid-State Electron. 16, 365 (1973).

${ }^{17}$ D. L. Scharfetter, Solid-State Electron. 8, 299 (1965).

${ }^{18}$ M. A. Green and J. Shewchun, Solid-State Electron. 16, 1141 (1973).

${ }^{19}$ M. A. Green and J. Shewchun, J. Appl. Phys. 46, 5185 (1975).

${ }^{20}$ P. Blood and J. W. Orton, Techniques of Physics 14: The Electrical Characterization of Semiconductors: Majority Carriers and Electron States (Academic, New York, 1992).
${ }^{21}$ C. Caso et al., Eur. Phys. J. C3, 1 (1998).

${ }^{22}$ E. H. Nicollian and H. Goetzberger, Bell Syst. Tech. J. 46, 1055 (1967).

${ }^{23}$ D. M. Taylor and H. L. Gomes, J. Phys. D 28, 2554 (1995).

${ }^{24}$ I. Balberg, J. Appl. Phys. 58, 2603 (1985).

${ }^{25}$ P. Stallinga, H. L. Gomes, G. W. Jones, and D. M. Taylor, Acta Phys. Pol. A 94, 545 (1998).

${ }^{26}$ H. Antoniadis, B. R. Hsieh, M. A. Abkowitz, S. A. Jenekhe, and M. Stolka, Synth. Met. 62, 265 (1994).

${ }^{27}$ J. Santamaria, G. Gonzales Diaz, E. Iborra, I. Martil, and F. SanchezQuesada, J. Appl. Phys. 65, 3236 (1989).

${ }^{28}$ C. H. Champness and J. Pan, Can. J. Phys. 66, 168 (1988).

${ }^{29}$ T. Misawa, J. Phys. Soc. Jpn. 12, 882 (1957).

${ }^{30}$ H. L. Gomes, Ph.D. thesis, University of Wales, Bangor, 1993.

${ }^{31}$ D. V. Lang, J. Appl. Phys. 45, 3023 (1974).

${ }^{32}$ E. F. Ferrari, M. Koehler, and I. A. Hümmelgen, Phys. Rev. B 55, 9590 (1997).

${ }^{33}$ P. Omling, E. R. Weber, L. Montelius, H. Alexander, and J. Michel, Phys. Rev. B 32, 6571 (1985) 